\begin{tabular}{|c|l|}
\hline Title & A method on strain measurement of HAP in cortical bone from diffusive profile of X-ray diffraction \\
\hline Author(s) & Fujisakia, Kazuhiro; Tadano, Shigeru; Sasaki, Naoki \\
\hline Citation & $\begin{array}{l}\text { Journal of Biomechanics, 39(3), 579-586 } \\
\text { https://doi.org/10.1016/.jbiomech.2005.01.001 }\end{array}$ \\
\hline Issue Date & 2006 \\
\hline Doc URL & http://hdl.handle.net/2115/4862 \\
\hline Type & article (author version) \\
\hline File Information & JB39-3.pdf \\
\hline
\end{tabular}

Instructions for use 


\title{
A METHOD ON STRAIN MEASUREMENT OF HAP IN CORTICAL BONE FROM DIFFUSIVE PROFILE OF X-RAY DIFFRACTION
}

\author{
Kazuhiro FUJISAKI ${ }^{1}$, Shigeru TADANO ${ }^{2}$ and Naoki SASAKI ${ }^{3}$
}

${ }^{1}$ Doctor Course Student, Division of Mechanical Science, Graduate School of Engineering, Hokkaido University, 060-8628, Japan

${ }^{2}$ Division of Mechanical Science, Graduate School of Engineering, Hokkaido University, 060-8628, Japan

${ }^{3}$ Division of Biological Sciences, Graduate School of Science, Hokkaido University, 060-0810, Japan

Corresponding author:

Shigeru TADANO

Professor, Division of Mechanical Science, Graduate School of Engineering, Hokkaido University, N13 W8, Kita-ku, Sapporo, 060-8628, Japan

TEL \& FAX: +81-11-706-6405, E-mail: tadano@eng.hokudai.ac.jp

Keywords:

Biomechanics, X-ray Diffraction, Cortical Bone, Hydroxyapatite, Strain Measurement

Manuscript type: Original Articles

Running title: Strain Measurement of HAp, Fujisaki et al.

Word count: 3072 words (Introduction through Concluding Remarks) 


\section{ABSTRACT}

Bone tissue is a composite material composed of hydroxyapatite (HAp) and collagen matrix. As HAp is a crystalline structure, an X-ray diffraction method is available to measure the lattice strain of HAp crystals. However, mineral particles of HAp in bone have much lower crystallinity than usual crystalline materials, which show a diffusive intensity profile of X-ray diffraction. It is not easy to determine quantitatively an infinitesimal strain of HAp from the peak position of diffusive profile. In order to improve the accuracy of strain measurement of HAp in bone tissue and to obtain reproducible results, this paper proposes an X-ray diffraction method applied to a diffusive profile for low crystallinity. This method is to estimate the lattice strain of HAp using not a peak position but a whole diffraction profile. In this experiment, a strip specimen of $28 \times 8 \times 2 \mathrm{~mm}$ was made from bone axial, outside circumferential and cross sectional circumferential region in the cortical bone of bovine femur. The X-ray diffraction measurements were carried out before and after applying an external load. As a result, the precision of strain measurement was much improved by this method. Although a constant value of macroscopic strain was applied in the specimen, the lattice strain had a lower value than the macroscopic strain and had a different value in each specimen.

Keywords:

Biomechanics, X-ray Diffraction, Cortical Bone, Hydroxyapatite, Strain Measurement 


\section{INTRODUCTION}

Bone tissue is often regarded as a composite material composed of hydroxyapatite (HAp) like mineral particles and collagen matrix in a microscopic scale. Because mineral particles are much stiffer than the matrix made of collagen fibers, the bone stiffness is influenced considerably by a mineral HAp in bone tissue. The deformation measurements of HAp particles under external loading are much important to understand the mechanical properties of bone in a microscopic scale. As HAp is a crystalline structure, an X-ray diffraction method is available to measure the lattice strain of HAp crystals.

A non-destructive and non-invasive method is desirable to investigate the stress or strain state of bone tissue in vivo. Cheng et al. (1995) have used the elastic wave propagation for measuring the elastic modulus of human tibia. The method has been widely used for determining the elastic modulus non-destructively. An X-ray diffraction method is also used to measure the stress or strain non-destructively in engineering and industrial field. However, few researches have applied the X-ray diffraction to the strain measurement of living bone tissue even in vitro. Tanaka et al. (1995) have reported the X-ray elastic constants for bone axial direction of compact bone by using characteristic X-rays. Tadano and Todoh et al. (1999, 2000) have confirmed that the X-ray diffraction method was effective to measure the stress in bone tissue and reported the existence of residual stress or strain in intact bone using polychromatic X-rays. However, mineral particles of HAp have much lower crystallinity than usual crystalline materials (Raquel, 1981; Matsushima et al., 1986). It is difficult to determine quantitatively a lattice strain in HAp from a peak position of a diffusive diffraction profile due to low crystallinity.

Borsato and Sasaki (1997) have reported a strain measurement for HAp particles at the bone axial direction under tensile loading, using a peak position of a diffracted intensity 
profile of X-rays. The lattice strain was obtained from only (004) lattice planes of HAp crystals. The (004) or (00l) pole are mainly oriented to the bone axial direction in long bone and cannot be used for the strain measurements in the other directions.

This paper presents a novel method to measure a lattice strain of HAp by using complex planes including the (211) planes that are not oriented to the bone axis. The method can be used to even a diffuse profile obtained from HAp crystals with low crystallinity. The lattice strain of HAp was estimated using not a peak position but a whole profile of diffracted intensity. The strip shaped specimens of $28 \times 8 \times 2 \mathrm{~mm}$ in size were cut from cortical bone in a shaft of bovine femur. While being loaded in four-point bending, the axial, outside circumferential and cross-sectional circumferential specimens were irradiated with X-rays.

\section{STRAIN MEASUREMENTS USING X-RAY DIFFRACTION}

\subsection{X-ray Diffraction Method}

Figure 1 shows the schematic X-ray diffraction phenomena in a crystalline material. When X-rays were irradiated to a crystalline specimen, diffraction of X-rays occurs in the specific direction by scattered X-rays from each atom. A beam path of diffracted X-rays relates to an interplanar spacing at a specific lattice plane $(h k l)$ in a crystal. Using characteristic X-rays with a definite wavelength $\lambda$, a diffracted angle $\theta$ can be obtained from a peak position of the diffracted intensity of X-rays. A fundamental equation for X-ray diffraction phenomena is called Bragg's law, and expressed as equation (1), where $d$ is the width of an interplanar spacing between adjacent lattice planes.

$$
2 d \sin \theta=\lambda
$$


The Bragg's law relates the diffraction angle $\theta$ to the interplanar spacing $d$. The lattice strain $\varepsilon$ in a lattice plane $(h k l)$ is defined as the ratio of interplanar spacing $d_{0}$ at the non-strained state and $d$ at the strained state of the same specimen. This relation is described by equation (2), where $\theta_{0}$ and $\theta$ are diffraction angles of non-strained state and strained state, respectively.

$$
\varepsilon=\frac{d-d_{0}}{d_{0}}=\frac{\sin \theta_{0}-\sin \theta}{\sin \theta}
$$

The coordinate system of bone specimen was defined as shown in Figure 2. The $x$-axis was taken parallel to the longitudinal axis of the strip shaped specimen $(28 \times 8 \times 2 \mathrm{~mm})$, and the $z$-axis was the perpendicular direction to the specimen plane. Because the incident and the diffracted beams run along the same X-ray path plane that is not parallel to $x$-axis, the strain $\varepsilon_{x}$ is not measured directly in this system. However, the value of $\varepsilon_{x}$ can be computed from some measurable strains in the other directions. Using the strain $\varepsilon_{\psi}$ on the plane with an angle $\psi$ inclined from the z-axis and principal strain components $\varepsilon_{1}$, $\varepsilon_{2}$ and $\varepsilon_{3}$, the strain $\varepsilon_{X}$ is represented by equation (3), where $\varepsilon_{3}$ is equal to $\varepsilon_{z}$.

$$
\varepsilon_{\psi}=\varepsilon_{1} \cos ^{2} \varphi \cdot \sin ^{2} \psi+\varepsilon_{2} \sin ^{2} \varphi \cdot \sin ^{2} \psi+\varepsilon_{3} \cos ^{2} \psi
$$

On the other hand, the strain $\varepsilon_{x}$ is represented as equation (4), where $\varphi$ is the angle of principal direction for $\varepsilon_{1}$ and $\varepsilon_{2}$.

$$
\varepsilon_{x}=\varepsilon_{1} \cos ^{2} \varphi+\varepsilon_{2} \sin ^{2} \varphi
$$


Eliminating $\varepsilon_{1}$ and $\varepsilon_{2}$ from equations (3) and (4), the strain $\varepsilon_{x}$ can be derived as equation (5).

$$
\varepsilon_{x}=\varepsilon_{\psi} \cdot \frac{1}{\sin ^{2} \psi}+\varepsilon_{z}\left(1-\frac{1}{\sin ^{2} \psi}\right)
$$

When both strain $\varepsilon_{\psi}$ and $\varepsilon_{z}$ are measured by the X-ray diffraction method, the strain $\varepsilon_{x}$ can be obtained from equation (5). In this measurement, the angle $\psi$ was selected as constant values of $40^{\circ}$ and $0^{\circ}$. When the angle $\psi$ is $0^{\circ}$, $\varepsilon_{\psi}$ is equal to $\varepsilon_{z}$.

\subsection{Optimal Strain Search from Intensity Profile of X-ray Diffraction}

Figure 3 shows a schematic intensity-angle profile of X-ray diffraction. In a highly crystallized material, a diffracted angle $\theta$ can be obtained easily from a peak position of the sharp profile according to the lattice spacing ( $h k l)$. The HAp structure in living bone has much small degree of crystallinity than metals such as steel. The profile of HAp is diffusive over a relating wide $2 \theta$ range. In this case, it is difficult to determine precisely a peak position.

The authors are proposing here a novel method to estimate the lattice strain with high reliability even for low crystallinity materials such as HAp particles in bone tissue. This method is to calculate the lattice strain using not a peak position but a whole diffraction profile. Figure 4 shows the relationship between intensity and diffracted angle of non-strained and strained state profile. The strained profile after tensile deforming moves to a smaller angle direction because of increasing the interplanar spacing between lattice planes. Not only a peak position but also every point on the non-strained state profile is assumed to be shifted by a strain value. The profile is divided into $n$ regions. Every value $\left(2 \theta^{0}{ }_{i}, I_{i}^{0}\right)$ on the profile 
was assumed to shift to the strained value $\left(2 \theta^{e}{ }_{i}, I_{i}^{e}\right)$, where the values were related by the follow equation at a constant value of strain $\varepsilon$. This equation was derived from equations (1) and (2), where $I$ is diffracted intensity and assumed no change under straining the specimen.

$$
\begin{aligned}
2 \theta_{i}^{e} & =2 \sin ^{-1}\left\{\frac{\sin \left(2 \theta_{i}^{0} / 2\right)}{\varepsilon+1}\right\} \\
I_{i}^{e} & =I_{i}^{0} \quad(i=1,2,3, \cdots, n)
\end{aligned}
$$

The strained values calculated by equation (6) are made to coincide with the points $\left(2 \theta^{e}{ }_{i}, I_{i}^{S}\right)$ on the strained state profile measured by the experiment. The deviation of the calculated values from the measured values is defined as the value $e$. When the value $e$ takes the minimum, an optimal strain $\varepsilon$ can be found in equation (7).

$$
\frac{\partial e}{\partial \varepsilon}=\frac{\partial\left\{\sum_{i=1}^{n}\left(I_{i}^{s}-I_{i}^{0}\right)^{2}\right\}}{\partial \varepsilon}=0
$$

\section{Experimental Procedure}

\subsection{Specimens}

In this experiment, the condition of every specimen was unified according to the following procedure. The fresh bovine femur of 36 month-old male was frozen at -25 deg.C prior to experiments. After thawing out with 24 hours at room temperature (20 \pm 3 deg.C and relative humidity of $70 \%$ ), the strip shaped specimens of $28 \times 8 \times 2 \mathrm{~mm}$ in size were cut from cortical bone of a shaft of bovine, as shown in Figure 5. Three types of specimen were 
prepared as shown in Figure 6. The longitudinal axis of specimen was aligned to the bone axial (specimen A), the outside circumferential (specimen B) and the cross sectional circumferential direction (specimen C). Every specimen was washed by an ultrasonic bath in physiological saline to eliminate undesired shavings. After a surface of specimen was dried out while 24 hours at room temperature, a strain-gage was glued to an opposite surface to that for X-ray irradiation. The experimental condition was kept at $20 \mathrm{deg}$. $\mathrm{C}$ and relative humidity of $70 \%$ to keep a constant degree of dryness of the specimen as possible during the X-ray measurement.

The bone specimen was applied to bending load by a four-points bending device shown in Figure 7. Using the device, the elastic modulus $E$ is measured as another test, which is derived by equation (8),

$$
E=\frac{3\left(l-l_{1}\right)}{2 b t^{2}} \cdot \frac{2 W}{\varepsilon}
$$

In above equation, each symbol of $l, l_{1}, 2 W, d$ or $t$ means supporting distance, loading distance, applied load, width or thickness of specimen, respectively.

\subsection{X-ray System}

Figure 8 shows the X-ray diffraction system (RINT2000, Rigaku Co., Japan) used in this experiment. The characteristic X-rays (Co-K $\alpha$, the wavelength $\lambda=0.1789 \mathrm{~nm}$ ) were generated from a Co target. Diffracted X-rays from a specimen were measured as an intensity-angle profile by a scintillation counter moving on the path of $2 \theta$ angle from $25^{\circ}$ to $50^{\circ}$. Detailed X-ray conditions were listed in Table 1. During the X-ray irradiation, a bending load was applied to the specimen using the four-point bending device of Figure 7. In order to obtain the 
lattice strain components $\varepsilon_{\psi}$ and $\varepsilon_{z}$, the X-ray diffraction profiles have to be measured under both non-strained state and strained state at both angles of $\psi=40^{\circ}$ and $0^{\circ}$. In this experiment, to focus reproducibility of the X-ray measurement, lattice strain was obtained by five measurements at the same loading condition.

\section{RESULTS}

The elastic modulus of each specimen was obtained as the mean value of five measurements under room temperature of 20 deg. C and humidity of $70 \%$. The elastic modulus showed the value of $24.6 \pm 1.0 \mathrm{GPa}$ (mean \pm S.D.) for specimen A, $15.5 \pm 0.6 \mathrm{GPa}$ for specimen B and 13.4 $\pm 0.5 \mathrm{GPa}$ for specimen C.

Figure 9 shows the X-ray diffraction profiles of the bone specimens and artificial HAp particles, which was obtained by scanning from $2 \theta=25^{\circ}$ to $50^{\circ}$ under no deformation. . The profile of artificial HAp particles showed high and obvious intensity peaks of (002), (211), (112), (300) and (310) planes. This is a typical for high crystallinity. In case of the bone specimen, the maximum peak is confirmed in the vicinity of (211) plane, while the profile has a diffusive and obscure peak of (211) because of including (112) and (300) peaks.

The strain is calculated from the X-ray diffraction profiles at non-strained state and strained. Figure 10 expresses the process of peak search from the profile. The complex profile including (211) plane is enlarged and shown in this figure. The diffusive profile was smoothed by the simple moving average using 71 data points in all 1001 data from $2 \theta=35^{\circ}$ to $40^{\circ}$. The background intensity was removed as a quadrilateral area including the both ends of a referenced profile region. In general, the strain is calculated from the peak position of diffracted angle $2 \theta$ by using the peak-top method or the FWHM (Full Width of Half 
Maximum Intensity) method. In the peak-top method, a diffracted angle $2 \theta$ is determined directly from the peak position of maximum intensity. In the FWHM method, a diffracted angle $2 \theta$ is determined as an angle of centre position of the width of profile at a half value of maximum intensity $(\mathrm{H} / 2)$. On the other hand, the method proposed in this work uses not a peak position but a whole diffraction profile.

Figure 11 shows an average value of the measured strains $\varepsilon_{\psi}, \varepsilon_{z}$ and a standard deviation calculated using this method. The strain $\varepsilon_{X}$ was derived from strains $\varepsilon_{\psi}$ and $\varepsilon_{z}$ using equation (5), where the angle $\psi$ was selected as $40^{\circ}$. When the specimen was deformed at a constant strain of $1000 \times 10^{-6}$ that was measured macroscopically by a strain gage, the strain $\varepsilon_{x}$ was $861 \pm 101 \times 10^{-6}$ (mean \pm S.D.) for the specimen A, $607 \pm 61 \times 10^{-6}$ for the specimen $B$ and $514 \pm 123 \times 10^{-6}$ for the specimen C.

Table 2 lists the experimental condition and the results of the elastic modulus $E_{x}$, the bending load $2 W$, the surface strain $\sigma_{x}$ and the lattice strain $\varepsilon_{x}$ for each specimen. The application of a constant macroscopic strain of $1000 \times 10^{-6}$ to each specimen corresponds with the different bending load of $44.2 \pm 1.8 \mathrm{~N}$ for the specimen $\mathrm{A}, 28.6 \pm 1.1 \mathrm{~N}$ for the specimen $\mathrm{B}$ and 24.2 $\pm 0.9 \mathrm{~N}$ for the specimen $\mathrm{C}$. The surface stress $\sigma_{x}$ in each specimen was 24.6 \pm 1.0 , 15.5 \pm 0.6 and $13.4 \pm 0.5 \mathrm{MPa}$ for specimen A, B and C, respectively. When the constant strain was applied to each specimen, the lattice strain of HAp crystals was a clearly higher value for the specimen with higher elastic modulus than the specimen with the lower elastic modulus. The elastic modulus and lattice strain were obtained in descending order of bone axial, outside circumferential and cross sectional circumferential region of a bovine shaft.

To confirm the applicability of the present method, the strain $\varepsilon_{X}$ was also calculated by the Peak-top method and the FWHM method. The result is illustrated in Figure 12. In comparison with the value of standard deviation of these methods, the method proposed in 
this work showed the smallest range of standard deviation. Therefore, this method is effective to measure the lattice strain of HAp in bone tissue.

\section{DISCUSSION}

The cortical region of a long bone is well known to be an anisotropic structure (Wainwright et al., 1976). The human femur consisting of Haversian bone has been considered to be transversely isotropic (Van Buskirk et al.,1981). Pithioux et al. (2002) have measured non-destructively an anisotropic elastic modulus of bovine cortical bone using the ultrasonic wave propagation and estimated the elastic constants as an orthotropic for lamellar structure of bovine femur. A significant difference in elastic moduli was recognized between bone axial and circumferential direction in cortical bone. Rho et al. (2002) have also performed the nano-indentation tests at an osteon level for the estimation of the elastic modulus of bone tissue. The deformation measurements of HAp particles under external loading are much important to understand the mechanical properties of bone in a microscopic scale. In this work, the anisotropic property of bone in microscopic scale was investigated on the relationship between macroscopic deformation and microscopic strain of HAp crystals. It was clear that the lattice strain was a lower value than macroscopic strain and was much different in each specimen even if applying a constant macroscopic strain.

In this work, in order to be clear the relationship between elastic modulus of specimen and lattice strain of HAp crystals, we selected three specimens with obviously different modulus in the same shaft of bovine femur. Although one specimen was used for each location, not only the elastic modulus but the lattice strain were obtained in descending order of bone axial, outside circumferential, cross sectional circumferential specimen. In general, 
the elastic modulus of bone is considered to be originated from a force bearing serial portion of HAp mineral particle. Such a mechanical structure can be attributed to preferred orientation of bone mineral. These results are indicating that the lattice strain of HAp is influenced by the elastic modulus in the specimen. We could confirm these results because of improving the accuracy of measurement by this method.

As shown in Figure 9, the X-ray diffraction profile of bone tissue is diffusive with lower intensity at every peak positions. In crystallography, thus profile is caused by the magnitude of the uniform interplanar spacing of crystal lattice (Cullity and Stock, 2001). The distribution of intensity profile is also known to be affected by the crystal orientation. The orientation of HAp c-axis adjusted the bone axis had already been investigated for the major part of $(00 l)$ planes (Sasaki and Sudoh, 1997). The profile of specimen C had especially a strong intensity at a diffracted angle from (002) planes. However, the (002) profile cannot be found in both specimens A and B. The peak of (211) plane including (112) and (300) was observed in every specimen. Because this method does not need to determine a peak position for strain calculation, the complex planes including (211) planes are available to measure a lattice strain of HAp. Therefore, this method can be used to measure the lattice strain of every location in the bone.

In this experiment, the information from X-ray penetration depth of incident beam has not been considered. The measured strain contains not only values of the surface but also of the inside of the specimen. At bending loading, the strain linearly decreases from a surface to the neutral axis of a specimen. The strain below the surface is not equal to surface strain. However, the penetrated X-ray of Co-K $\alpha$ decreases very rapidly as a function of depth, which is related to X-ray absorption number of constituent atoms of materials and its density (Koch et al., 1962). Therefore, the strain inside the specimen is regarded as negligibly small. The measured lattice strain can be regarded as the strain of HAp in the surface. 
The relative humidity of $70 \%$ indicates that the specimen was equilibrated with laboratory atmosphere. This humidity condition was needed for the gluing of a strain gage on the specimen surface. The condition is different from the physiological state and the mechanical properties of such a bone were different from that measured in physiological saline (Sasaki et al., 1995). However, the aim of the present study is to present a novel technique to detect precisely a microscopic strain in bone as a response to externally applied force. For this purpose, we considered that the state of the specimen did not matter.

The structures of HAp crystals and collagen fibre or the shape of HAp particles remain many questions still under discussion (Sasaki et al., 2002). To determine the strain of HAp crystals in bone tissue under loading is important to understand the mechanical composite structure of HAp crystal and collagen fibre in bone. The method presented here to calculate the lattice strain from not a peak position but a whole shape of diffraction profile gave an advanced accuracy of strain measurement for bone tissue. In the future work, we will provide a better investigation to obtain anisotropic property of the bone tissue in microscopic level. If the lattice strain of HAp is related to macroscopic strain with anisotropic properties of bone tissue, it will be possible to obtain the macroscopic stress at tissue level non-invasively by X-rays. This work shows a possibility to measure the strain non-invasively and quantitatively in living bone.

\section{CONCLUDING REMARKS}

The lattice strain of HAp crystal could be obtained with high precision from a whole shape of diffracted intensity profile of X-ray diffraction by this method. The lattice strain was lower value than the macroscopic strain in every specimen and clearly higher value for the 
specimen with higher elastic modulus than the specimen with the lower elastic modulus under a constant macroscopic strain.

\section{ACKNOWLEDGEMENTS}

This work was supported by Grant-in-Aid for Scientific Research (B) (2), MEXT (No.16300143).

\section{REFERENCES}

Borsato, K. S., Sasaki, N., 1997. Measurement of partition of stress between mineral and collagen phases in bone using X-ray diffraction techniques, J. Biomechanics 30(9), 955-957.

Cheng, S., Timonen, J., Suominen, H., 1995. Elastic wave propagation in bone in vivo: Methodology, J. biomechanics 28(4), 471-478.

Cullity, B. D., Stock, S. R., 2001. Elements of X-ray diffraction, Prentice-Hall Inc. U. S. A., 167-184.

Koch, B., Macgillavry, C. H., Milledge, H. J., Koopmans, K., Rieck, G. D., Bacon, G. E., Absorption. In: Lonsdale, K. (Ed.), International tables for X-ray crystallography, vol. 3, The kynoch press, U. K., 157-200.

Matsushima, N., Tokita, M., Hikichi, K., 1986. X-ray determination of the crystallinity in bone tissue, Biochimica et Biophysica Acta 883, 574-579.

Pithioux, M., Lasaygues, P., Chabrand, P., 2002. An alternative ultrasonic method for measuring the elastic properties of cortical bone, J. Biomechanics 35, 961-968. 
Raquel, Z. L., 1981. Apatites in biological systems, Prg. Crystal Growth and Characterization of Materials 4, 1-45.

Rho, J. Y., Zioupos, P., Currey, J. D. and Pharr, G. M., 2002. Microstructural elasticity and regional heterogeneity in human femoral bone of various ages examined by nano-indentation, J. Biomechanics 35, 189-198.

Sasaki, N., Enyo, A., 1995. Viscoelastic properties of bone as a function of water content, J. Biomechanics 28, 809-815.

Sasaki, N., Sudoh, Y., 1997. X-ray pole figure analysis of apatite crystals and collagen molecules in bone, Calcif Tissue Int. 60, 361-367.

Sasaki, N., Tagami, A., Goto, T., Taniguchi, M., Nakata, M. and Hikichi, K., 2002. Atomic force microscopic studies on the structure of bovine femoral cortical bone at the collagen fibril-mineral level, J. Materials Science: Materials in Medicine 13, 333-337.

Tadano, S., Okoshi, T. and Shibano, J., 2000. Residual stress induced from bone structure and tissue in rabbit's tibia, Proc. of 10th Int. Conf. on Biomedical Eng., 529-530.

Tadano S. and Todoh M., 1999. Anisotropic residual stress measurements in compact bone using polychromatic X-ray diffraction, In: Pedersen, P. and Bendisøe, M. (Ed.), IUTAM Symposium on Synthesis in Bio Solid Mechanics, Klumer Academic Publications, Pordr Netherland, 139-159.

Tanaka, K., Akiniwa, Y., Suzuki, K. and Takezono, T., 1995. Mechanical anisotoropy and texture of bovine compact bone, Proc. of the Int. Symp. on Adv. Tech. in Exper. Mech., 213-218.

Todoh, M., Tadano S., Shibano J. and Ukai T., 2000. Polychromatic X-ray measurements of anisotropic residual stress in bovine femoral bone, JSME International Journal, Series C. 43-4, 795-801. 
Van Buskirk, W. C. and Ashman, R. B., 1981. The elastic moduli of bone, In: Cowin, S. C. (Ed), Mechanical properties of bone, ASME, U. S. A., 131-143.

Wainwright, S. A., Biggs, W. D., Currey, J. D. and Gosline, J. M., 1976. Structure of bone; Mechanical properties of bone, In: Mechanical design in organisms, Edward Arnold Ltd., U. K.,160-187. 


\section{Figure and Table Legends}

Fig. 1 The schematic X-ray diffraction phenomena in crystalline material model. Diffracted X-ray beam path is determined by interplanar spacing.

Fig. 2 For specific strain measurement, a coordinate system is defined on the surface of specimen; $x$-axis is aligned with loading direction, $z$-axis is perpendicular direction, an angle $\psi$ is defined as inclined direction from $z$ to $x$ direction.

Fig. 3 The X-ray diffraction profiles measured from high crystallinity material and low crystallinity material. The profile of HAp in bone tissue is diffusive on the whole because of low crystallinity.

Fig. 4 Strained state point estimated from non-strained state profile and compared to strain state profile. Difference between estimated point and measured point on the strained state profile was used for optimal strain evaluation.

Fig. 5 Strip specimen cut from bovine cortical bone was used to obtain macroscopic elastic modulus and microscopic lattice strain under bending deformation.

Fig. 6 Cutting location of bone specimens; shaft of bovine femur, 36 month-old of male. Longitudinal direction of each specimen A, B and C were aligned with bone axial, circumferential on the side and on cross sectional region, respectively 
Fig. 7 A four points bending system and the specimen setup. X-rays irradiate to tensile side and macroscopic strain was measured by strain-gage on compression side.

Fig. 8 X-ray measurement system (RINT2000, Rigaku Co.).

Fig.9 X-ray diffraction profiles of artificial HAp reference as high crystallinity and bone specimens. Representative peaks on the bone profiles occurred at each position of HAp typical diffraction angle from lattice plane $(h k l)$.

Fig. 10 The process of peak search from X-ray diffraction profile. The simple moving average 71 data points in all 1001 data. The background intensity was removed as a quadrilateral area including the both ends of a referenced profile region.

Fig. 11 Measured strain components at (211) plane and strain $\varepsilon_{x}$ calculated from equation (5). Measurement area is shallow depth from surface on the specimen deformed tensile 1000 micro strain by bending force.

Fig. 12 Lattice strain at (211) plane on the surface of the specimens calculated from some methods; peak top method and FWHM method are conventional method for strain calculation using peak position. The mean value and standard deviation in five times measurement for each specimen were indicated in this figure. The specimens were the bone axial (A), the outside circumferential (B) and the cross sectional circumferential direction (C). 
Table 1 X-rays generated from Co target in RINT2000 system and diffracted X-rays were measured by a scintillation counter under listed condition. To measure $\varepsilon_{z}$ and $\varepsilon_{\psi}$, inclined angles were selected $0^{\circ}$ and $40^{\circ} \mathrm{deg}$.

Table 2 Elastic modulus $E_{x}$, bending load $2 W$, surface strain value $\sigma_{x}$, lattice strain $\varepsilon_{x}$ under a constant value of 1000 micro strain. These values were represented as (mean \pm S.D.). 
Figure 1

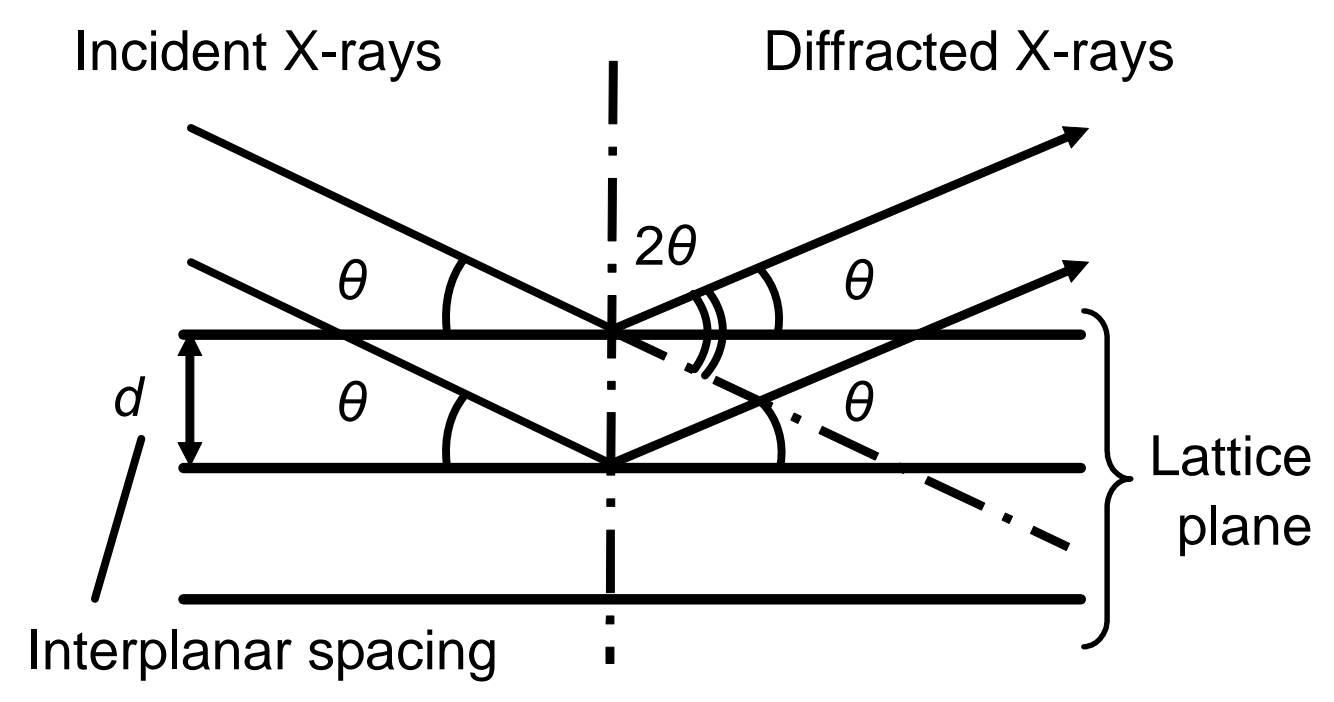


Figure 2

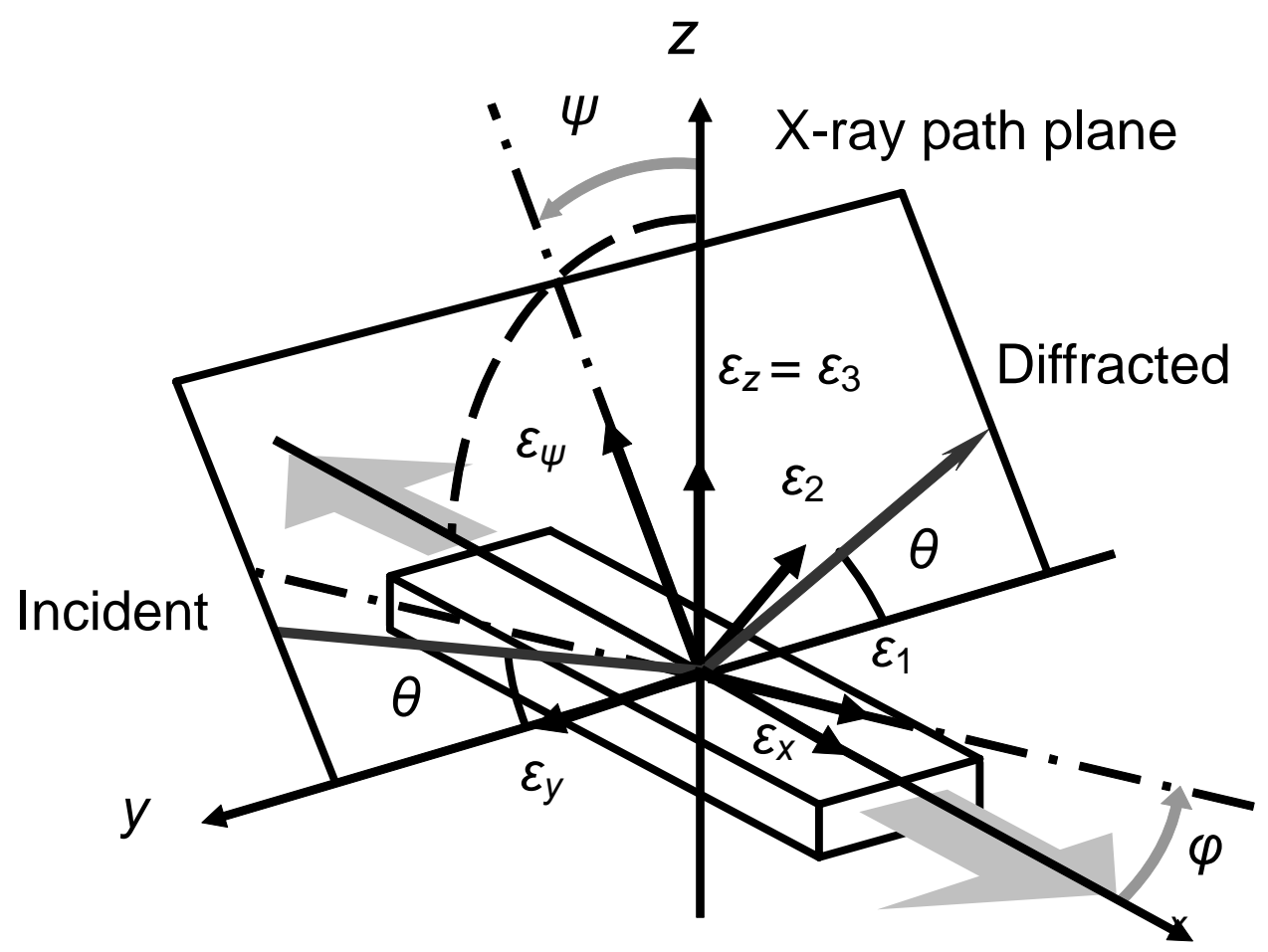


Figure 3

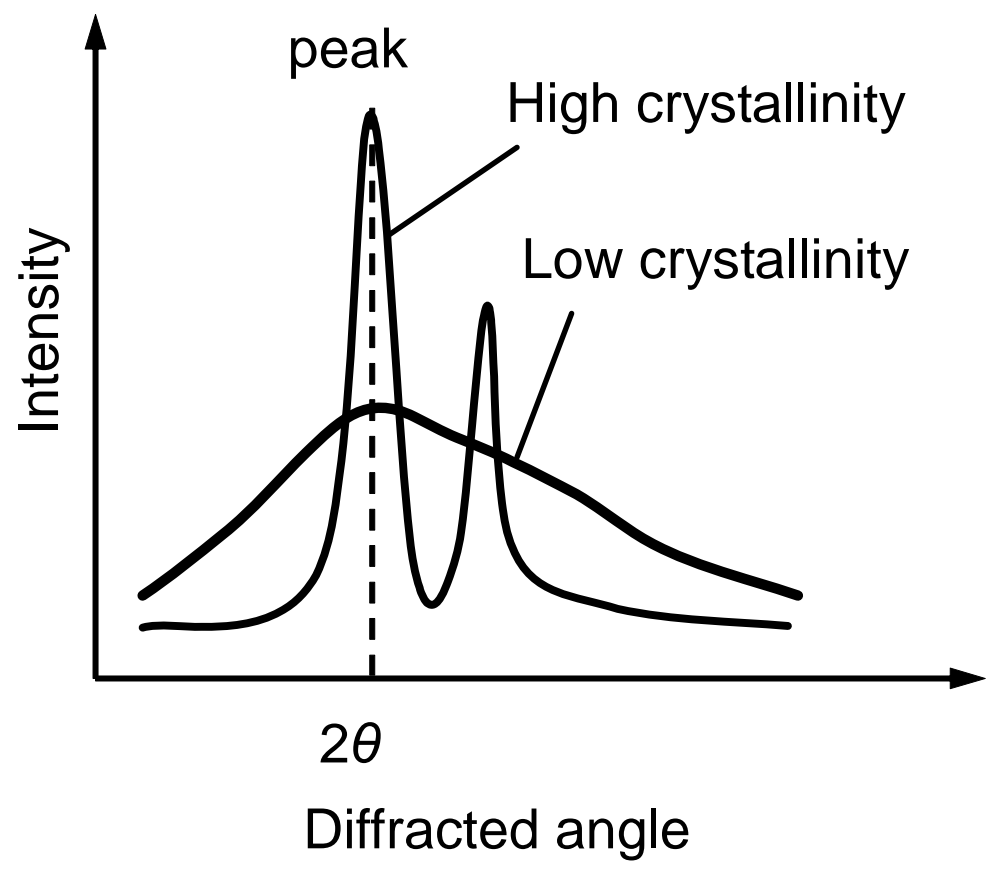


Figure 4

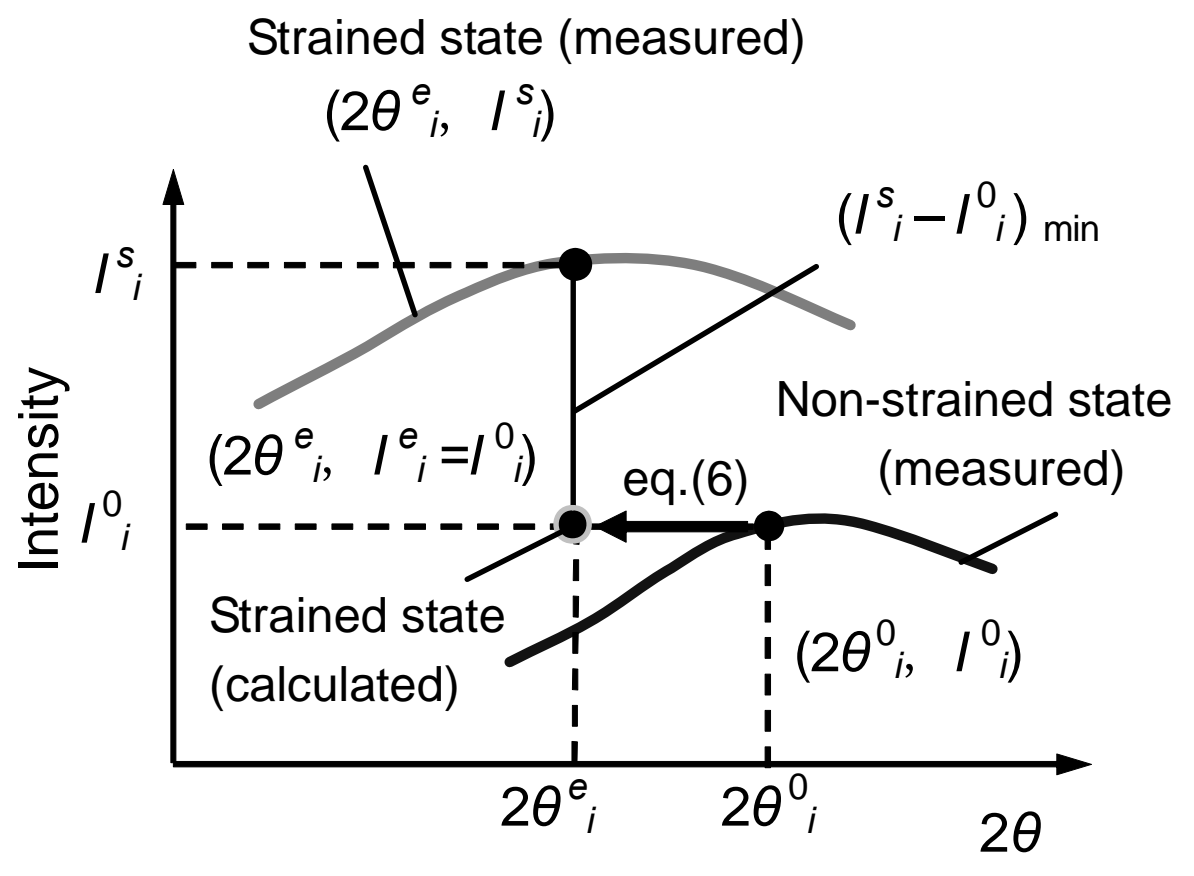


Figure 5

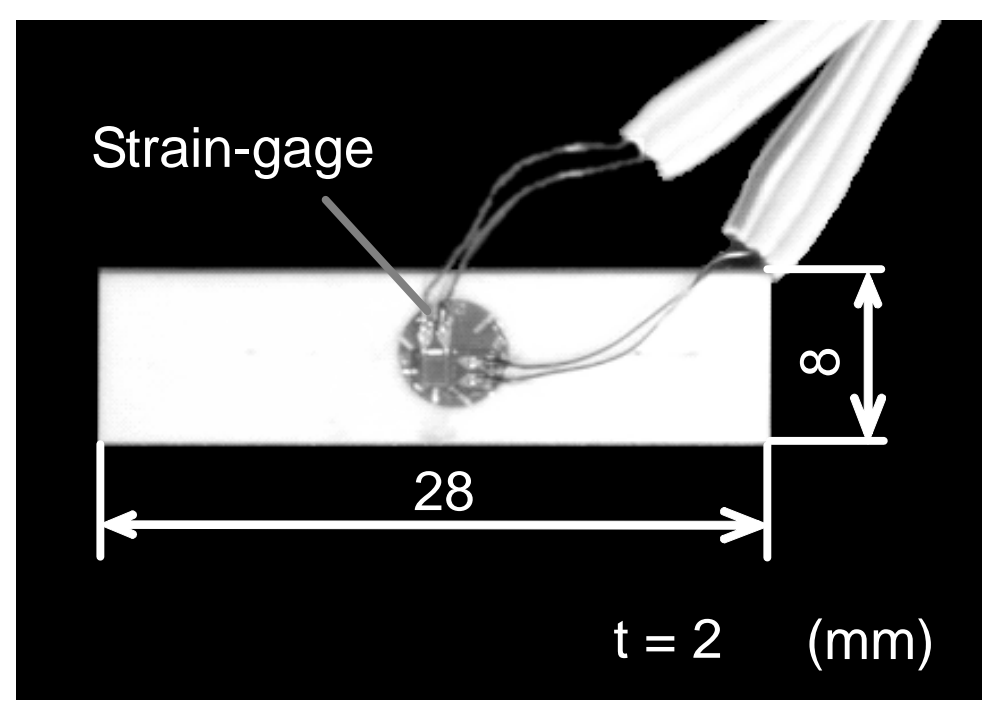


Figure 6

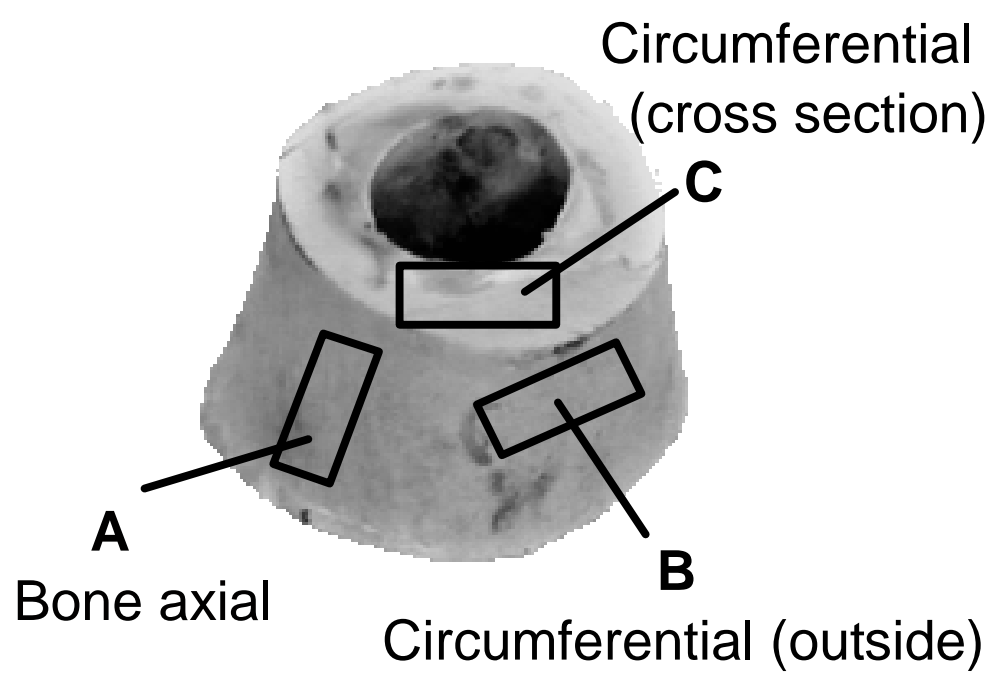


Figure 7

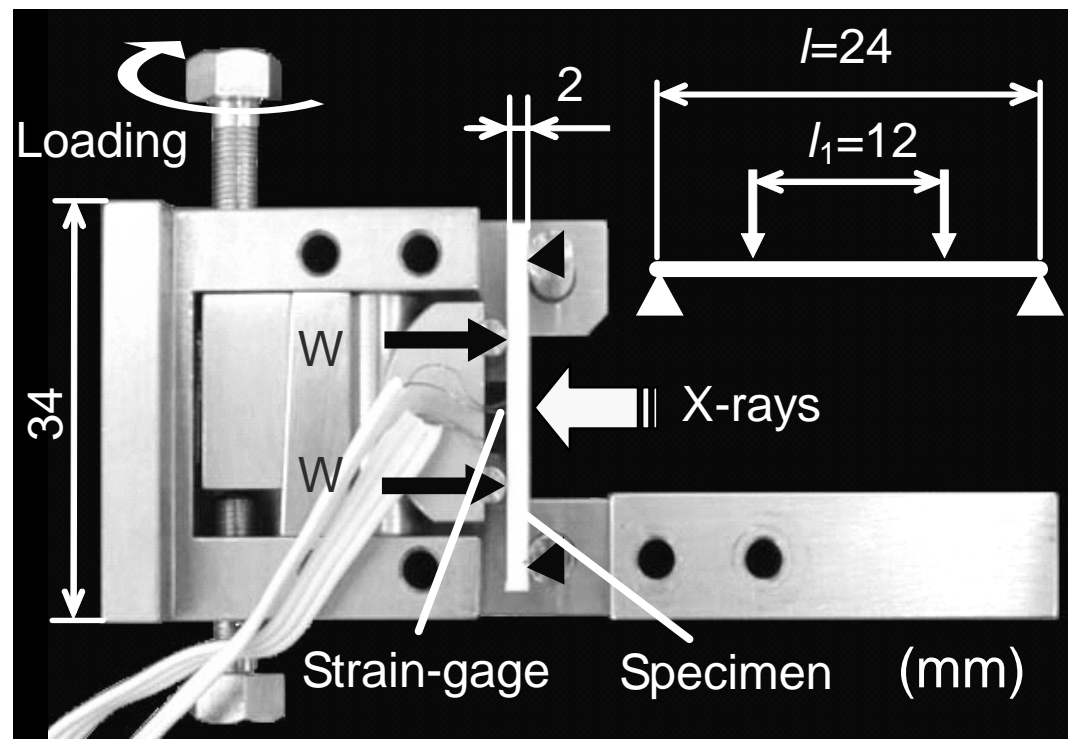


Figure 8

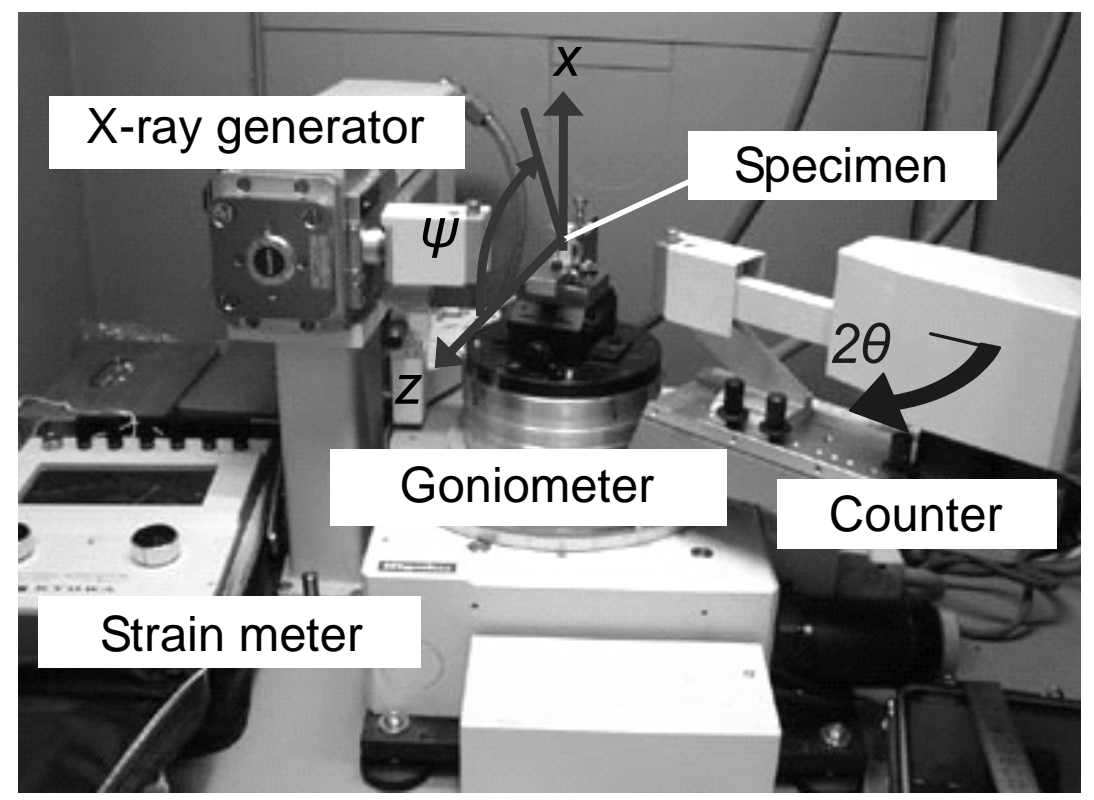


Figure 9

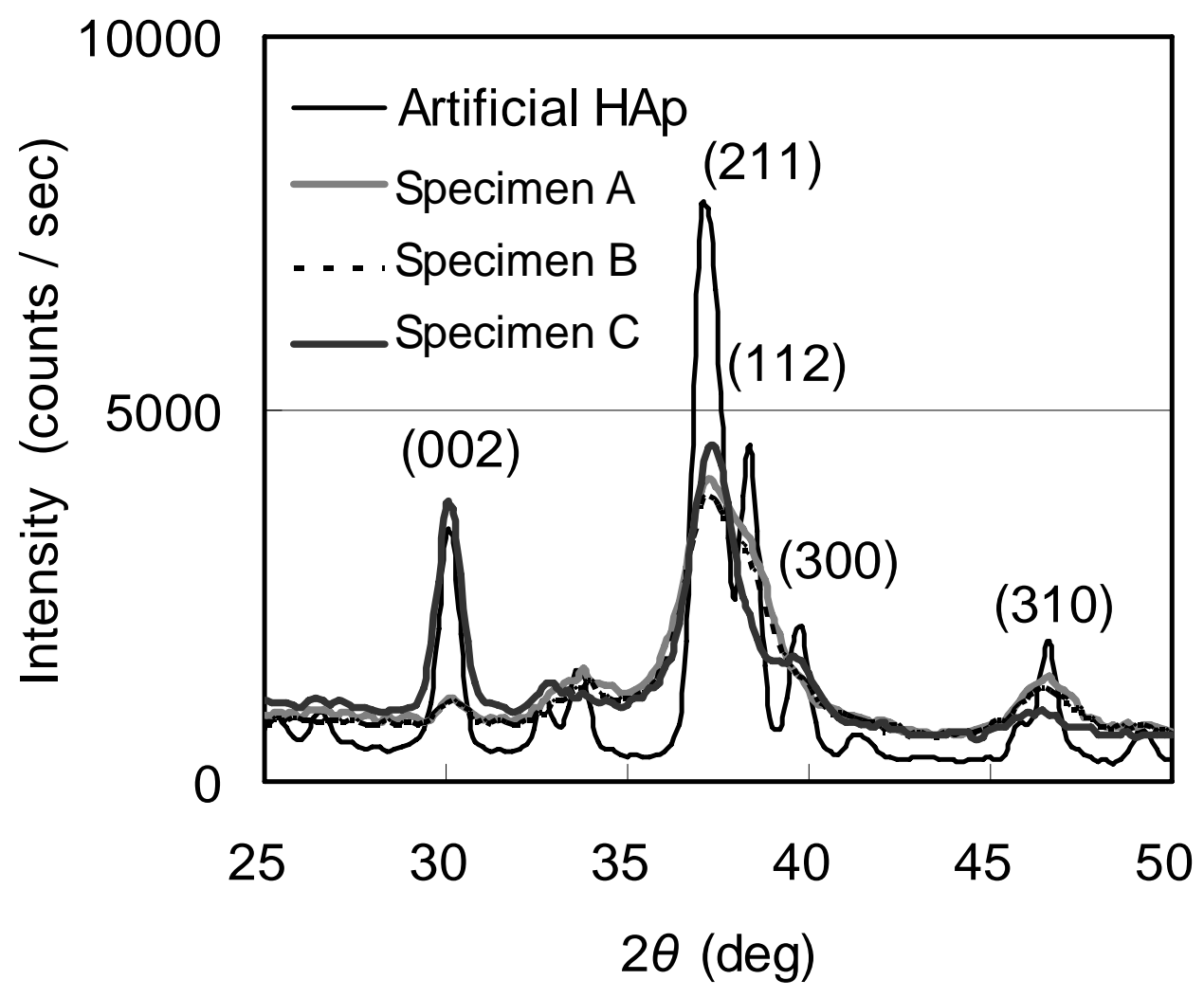


Figure 10

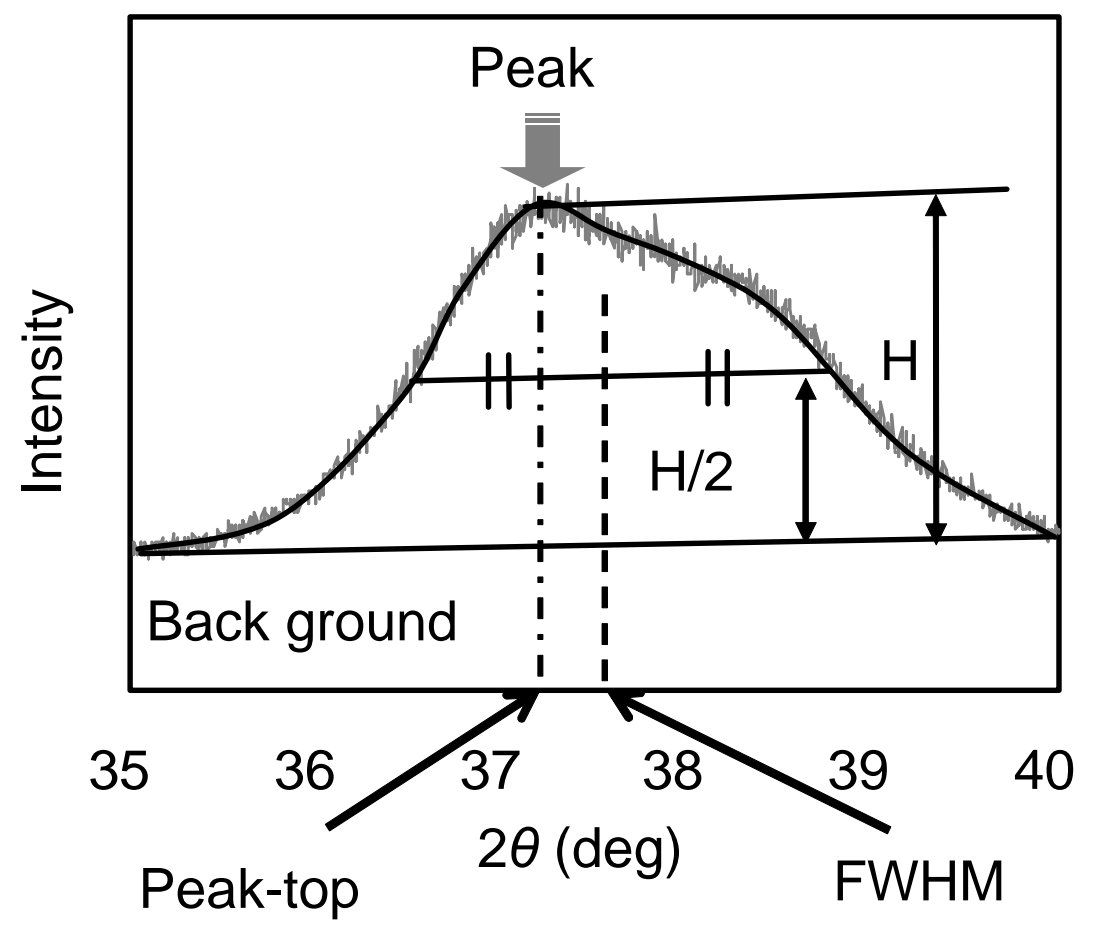


Figure 11

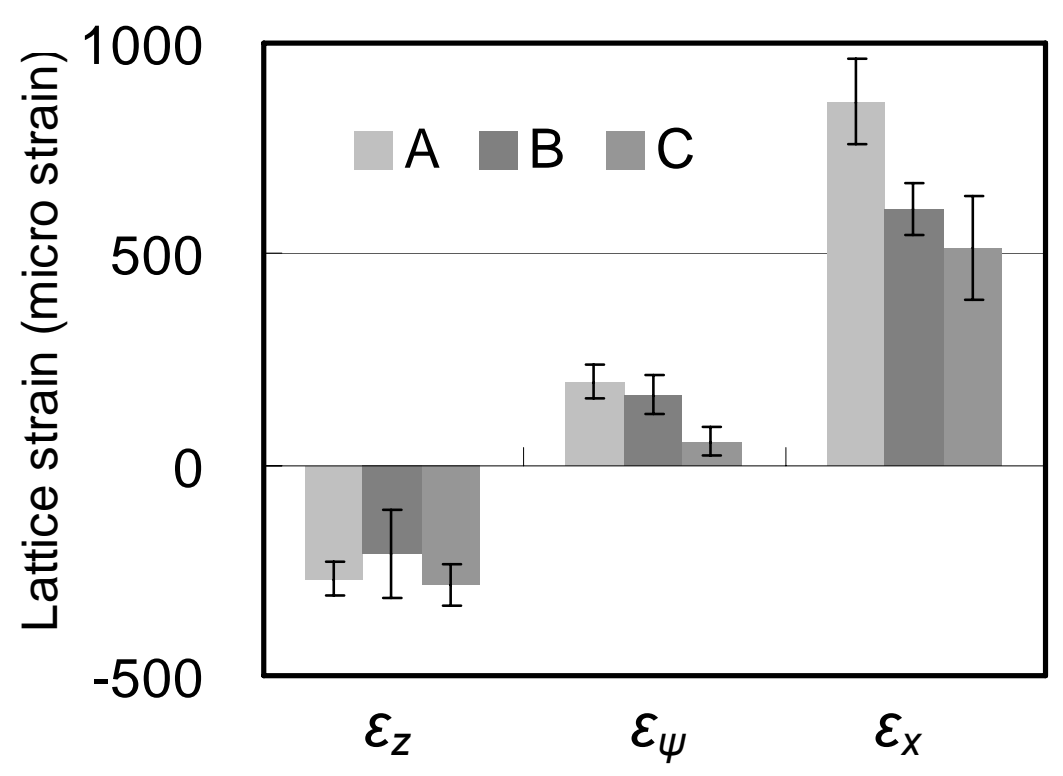


Figure 12

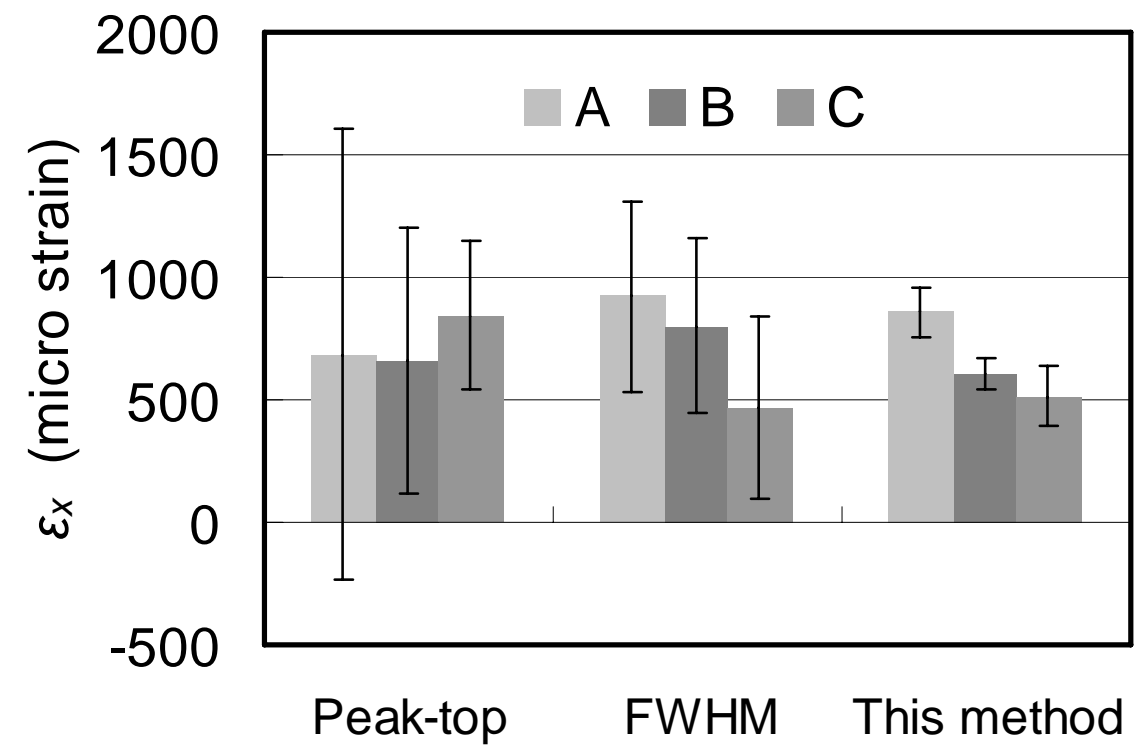


Table 1

\begin{tabular}{lcc}
\hline Target & & Co \\
Characteristic X-rays & & Co-K $\alpha$ \\
Wave length $\lambda$ & $(\mathrm{nm})$ & 0.1789 \\
Filter & & $\mathrm{Fe}$ \\
Tube voltage & $(\mathrm{kV})$ & 40 \\
Tube current & $(\mathrm{mA})$ & 40 \\
Measurement angle $2 \theta$ & $(\mathrm{deg})$ & $35 \sim 40$ \\
Scan speed & $(\mathrm{deg} / \mathrm{min})$ & 0.5 \\
Sampling width & $(\mathrm{deg})$ & 0.005 \\
Inclination angle $\psi$ & $(\mathrm{deg})$ & 0,40 \\
\hline
\end{tabular}


Table 2

\begin{tabular}{c|c|ccc}
\hline & A & B & C \\
\hline \multicolumn{2}{c|}{$E_{X}(\mathrm{GPa})$} & $24.6 \pm 1.0$ & $15.5 \pm 0.6$ & $13.4 \pm 0.5$ \\
\hline $2 W(\mathrm{~N})$ & $44.2 \pm 1.8$ & $28.6 \pm 1.1$ & $24.2 \pm 0.9$ \\
\hline \multirow{2}{*}{$\begin{array}{c}\sigma_{x}(\mathrm{MPa}) \\
\varepsilon_{x}\end{array}$} & Peak top & $686 \pm 918$ & $661 \pm 546$ & $844 \pm 305$ \\
\cline { 2 - 5 }$($ micro strain) & FWHM & $921 \pm 390$ & $803 \pm 353$ & $469 \pm 369$ \\
\cline { 2 - 5 } & This method & $861 \pm 101$ & $607 \pm 61$ & $514 \pm 123$ \\
\hline
\end{tabular}

